\title{
Influence of compound aescine gel on ultrastructure of vein infused mannitol and its mechanism
}

\author{
Jinhua Zhang ${ }^{1^{*}}$, Hefang Wang ${ }^{1}$, Yanling Gui ${ }^{2}$, Wei $\mathrm{Li}^{3}$, Wei Zhou ${ }^{4}$ \\ ${ }^{1}$ School of Nursing, Xinxiang Medical University, Xinxiang, China; ${ }^{*}$ Corresponding Author: zjh.warm@,163.com \\ ${ }^{2}$ First Affiliated Hospital of Xinxiang Medical University, Xinxiang, China \\ ${ }^{3}$ Sanquan Medical College, Xinxiang Medical University, Xinxiang, China \\ ${ }^{4}$ Huanggang Polytechnic College of Hubei Province, Huanggang, China
}

Received 27 December 2012; revised 3 February 2013; accepted 18 February 2013

Copyright (C) 2013 Jinhua Zhang et al. This is an open access article distributed under the Creative Commons Attribution License, which permits unrestricted use, distribution, and reproduction in any medium, provided the original work is properly cited.

\section{ABSTRACT}

Purpose: When hypertonic solution 20\% mannitol solution was injected into vein, inflammatory mediators and Mitogen-activated protein kinases activated by mannitol can directly induce the fading of vascular endothelial cell, which leads to phlebitis. The study aims to observe the influences of reparil-gel $\mathrm{N}$ coated at the proximal parts of the puncture point and basing on this along with infusing heated mannitol to veins to the injure and ultrastructure of veins which were infused the $20 \%$ mannitol solution by indwelling needle in vein. Methodology: There are 15 adult New Zealand rabbits. We randomly divided 24 ear veins of 12 adult New Zealand rabbits into Control group, Gelatum group, Gel heated group and injected $20 \%$ mannitol solution by vein detained needle in three groups. In Gelatum group, we coated the proximal end of the puncture point with a thin layer of compound aescine gel. Based on Gelatum group, we heated $20 \%$ mannitol solution to $35^{\circ} \mathrm{C}-37^{\circ} \mathrm{C}$ in Gel heated group. Then we observed the intravenous parts and took the veins of each group out to observe their morphology and ultrastructural after the second day of transfusion. 6 ear veins of the rest 3 rabbits as Health group weren't given any interventions, the veins were taken out to observe their morphology. Results: Comparison between Gelatum group and Gelatum heated group on vascular dilatation, Infiltration of inflammatory cell and Formation of thrombus had no significance, $P>0.05$, while the case was different for the comparison on injury of vascular wall, perivascular edema and perivascular hemorrhage, $P<$
0.05). The statistical significance exists between control group and Gelatum group and Gel heated group, $P<0.05$. It was observed under the electron microscope that, in control group, the membrane of endothelial cell peeled off and the mitochondria swelled and vacuolized. In Gelatum group, the membrane of endothelial cell was defective, the parts of the mitochondria were fuzzy, intercellular substance was slightly edematous. In Gel heated group, the mitochondria were clear and intercellular substance slightly swelled. It could be found that the function of phagocyte was complete. Conclusions: Compound aescine gel can prevent phlebitis or reduce the incidence of phlebitis. The combined intervention of coating with a thin layer of compound aescine gel and heating mannitol solution can produce better effect.

Keywords: Hyperosmotic Solution; Compound Aescine Gel; Phlebitis; Vein Detained Needle; Ultrastructure

\section{INTRODUCTION}

Venous indwelling trocar, which has an extensive application in clinical practice, can reduce patients' pain caused by repeated puncture and lower the labor intensity of nurses. The long-term infusing or inputting high concentrated or strongly pungent drugs through venous retention needles can disturb the homergy and function of vascular endothelial cell, which causes mechanical or chemical phlebitis, especially inflammatory mediators and Mitogen-activated protein kinases activated by mannitol can directly induce the fading of vascular endothelial cell, and intravascular devices-related phlebitis is 
also a significant problem [1]. It was estimated that 50\% $80 \%$ of patients with chemotherapy, annually, have different degree of phlebitis [2]. The occurrence of phlebitis not only increases the pain of patients, but also shortens the using time of venous indwelling trocar. At present, the domestic colleagues actively explore effective therapy of local phlebitis or injure of perivascular tissues caused by intravenous infusion but most of the research are clinical study instead of the research on the prevention of phlebitis caused by intravenous infusion. At home and abroad, few researches are conducted on the protective effects of compound aescine gel to phlebitis caused by intravenous infusion especially experiment study. This experiment aims to investigate the effects of compound aescine gel on the rabbits' ear veins that received the infusion of hyperosmotic solution through vein detained needle and provides theoretical basis for the clinical protection of intravenous infusion vessels through observing the pathological changes of the tested vascular ultrastructure by transmission electron microscopy.

$20 \%$ mannitol solution is a kind of dehydrant which is widely used in patients with high intracranial pressure and cerebral edema. It can reduce the high intracranial pressure and cerebral edema. However, as a hypertonic solution, long and repetitive infusion of mannitol can cause phlebitis. Nowadays, especially the wide application of vein detained needle to inject not only makes the needle lose its proper function, but also increases the incidence of phlebitis and the suffering of patients in clinics. As a compound, it has significant effect on resisting inflammation, anti-oozing and eliminating swelling in nature, compound aescine gel can restore the normal capillary permeability, increase the venous tone, and improve microcirculation. It is used to relieve pain and block the abnormal inflammatory response of the closed soft tissue injury in clinics. But the effects of compound aescine gel compound to prevent phlebitis caused by intravenous infusion need further study. And we probed into it.

\section{MATERIALS AND METHODS}

\subsection{Materials and Samples}

Experimental animals: 15 healthy New Zealand adult rabbits were selected, weight $2-2.5 \mathrm{~kg}$, male and female are unlimited. Exclusion standard: malformations and or damage of the veins of rabbit ears. Experimental drugs and main reagents: compound aescine gel (Reparil Gel), Dr Ma Pharmaceutical production, Germany, specifications: 100 grams/branch; $20 \%$ mannitol and normal saline and heparin saline (15 U/m1); 4\% glutaraldehyde fixation fluid and 25\% Urethane solution. Main instrument: Hitachi H-7500 transmission electron microscopy;
\# 24 venous retention needles produced by American BD company and microinfusion pump provided by laboratory of nursing school, Xinxiang medical university, etc.

\subsection{Grouping and Interventions}

The New Zealand adult rabbits qualified for the requirement were fed for three days to make them adaptive. We cleaned and removed the hair on the injection areas of the rabbits' double ears by using Sodium sulphide solution on the second days. We randomly selected 12 the rabbits, two ear margin veins of every rabbit were used at the same time, totally 24 veins. Then we randomly divided 24 ear margin veins into control group, Gelatum group, Gelatum and heated mannitol group (shortened form: Gel heated group). The Control group: we only injected $20 \%$ mannitol solutions into the ear margin vein by vein detained needle. The Gelatum group: we exteriorly coat the skin with a thin layer of compound aescine gel on the small area $(2-3 \mathrm{~cm}$ away from the puncture point; covering $3-4 \mathrm{~cm} \times 2 \mathrm{~cm}$ ) every time before injecting 20\% mannitol solution. The Gel heated group: on the basis of the outer coating gel, we heated $20 \%$ mannitol solution to $35^{\circ} \mathrm{C}-37^{\circ} \mathrm{C}[3,4]$ with constant temperature water-bath water box before injecting. Microinfusion pump was used in three groups to infuse $2.50 \mathrm{ml} / \mathrm{kg}$ (two ears used simultaneously) at the speed of $0.5 \mathrm{ml} / \mathrm{min}$ [5], twice a day for 2 days. And we used $10-15 \mathrm{ml}$ of normal saline both before and after the infusion of mannitol. After the injection, we sealed the introcans with $2.5 \mathrm{ml}$ heparin saline in positive pressure. We sterilized the rubber stoppers of the vein cap in lodophor, and then penetrated scalp needle into the vein cap when we infused the drug again. The rest 3 rabbits, 6 ear margin veins without any injection were studied as Health group. The experimental process was under way by specialist with strict aseptic operations.

\subsection{Specimen Preparation}

On the third days of the experiment, we randomly selected one rabbit from three groups respectively and anesthetized them with $25 \%$ Urethane solution, $3 \mathrm{ml} / \mathrm{kg}$ by intramuscular injection. We fixed the tested blood vessels with $4 \%$ glutaraldehyde perfusion for 10 minutes in the body, then took about $1 \mathrm{~mm} \times 1 \mathrm{~mm} \times 1 \mathrm{~mm}$ size vein samples from $1 \mathrm{~cm} \times 1 \mathrm{~cm}$ auricular tissues of the vein needle tip part $0.5 \mathrm{~cm}$ (proximal part of the heart) and put them into the $4 \%$ Glutaraldehyde solution in $4{ }^{\circ} \mathrm{C}$. We fixed the specimens with $1 \%$ osmium tetroxide acid for $1 \mathrm{~h}$ when specimens had been fixed in Glutaraldehyde liquid for $24 \mathrm{~h}$. Following the conventional procedure of electron microscopic specimens, we made specimens, cut them into slices with ultramicrotome, whose section 
thickness was $60 \mathrm{~nm}$, and then dyed them with acetic acid uranium saturated solution and folic acid lead. We observed the change of vascular ultrastructure with transmission electron microscopy of Hitachi H-7500 and took pictures. We put the rest of auricular tissues into $4 \%$ paraformaldehyde to fix, then made conventionally pathological section. After $24 \mathrm{~h}$, we gave them HE staining and observed them with Light microscope.

\subsection{Pathological Judgment Standard}

This research aims to judge the pathological changes of veins and perivascular tissues from six aspectsVascular wall, Perivascular edema, Infiltration of inflammatory cell, Vascular dilatation, Perivascular hemorrhage and Formation of thrombus. Each item of pathological injury degree is divided into: - (normal), + (minor), ++ (moderate), +++ (severe) 4 grades. Specific evaluation criteria are as follows: 1) the degree of vascular wall Injury: "-" normal, "+" damage extent accounts for $25 \%$ of vascular circumference, "++" damage extent accounts for $26 \%-50 \%$ of vascular circumference, " +++ " damage extent accounts for more than $50 \%$ of vascular circumference; 2) the degree of vascular dilatation: “_" normal, "+" vascular mild dilatation, "++" vascular moderate dilatation, " +++ " vascular severe dilatation; 3) the degree of inflammatory cell infiltration: "-" normal, “+” a few scattered inflammatory cells infiltration in veins or perivascular tissues, "++" many inflammatory cells infiltration, "+++" intensive inflammatory cells infiltration; 4) the degree of perivascular edema: "-" perivascular tissues without edema, "+" local tissues with slight edema, "++" edematous tissues showing cluster or flake, "+++" tissues with wild-ranging edema; 5) the degree of perivascular hemorrhage: "-" normal, "+" perivascular focal hemorrhage, "++" perivascular splinter hemorrhage, "+++" perivascular diffuse hemorrhage; 6) the degree of thrombus formation: "-" vascular lumen without thrombus, "+" thrombus volume in vascular lumen accounting for one third of vascular lumen area, "++" thrombus volume in vascular lumen accounting for two thirds of vascular lumen area, " +++ " thrombus volume in vascular lumen accounting for more than two thirds of vascular lumen area.

\section{STATISTICAL METHODS}

We used Statistical Package for the Social Sciences (SPSS) version 13.0 (Chicago, IL) for data entry and statistical analyses. We performed comparisons presented as the incidence of phlebitis and the injure degree of veins among groups by using Fisher probabilities and Kruskal-Wallis $\mathrm{H}$ test respectively. We consider a $P$ value $<0.05$ to be statistically significant.

\section{RESULTS}

\subsection{Light Microscopic Observation}

According to pathological judgment standard, the veins and perivascular tissues were normal to Health group (see Figure 1(a)). Comparing the Control group with the Healthy group, the Control group was found that injury of vascular wall was heavier, inflammatory cells infiltration was serious, severe vascular dilatation, vascular lumen perivascular edema and severe congestion occurred, a large of thrombus could be seen in vascular lumen (see Figure 1(b)). Slight vascular wall Injury happened to Gelatum group in which vascular was mild dilatation, scattered infiltration of inflammatory cells were few, local tissues had slight edema, perivascular tissues had no hemorrhage, no thrombus formed, but had a small amount of exudate (see Figure 1(c)). In the Gel heated group, vascular structures closed to normal; vessel wall had no injury; there were a small amount of infiltration of inflammatory cells, mildly vascular dilatation, and no thrombus formation; perivascular tissues had slight edema (see Figure 1(d)). The rate of phlebitis is $100 \%$ and very severe in Control group. And comparing the injury situation of ear margin veins and perivascular tissues in Control group with that of the Gelatum and the Gel heated group had statistical significance $(P<0.05)$. Comparison between Gelatum group and Gel heated group on Vascular dilatation, infiltration of inflammatory cell and Formation of thrombus had no significance $(P>$ $0.05)$, while the case was different for the comparison on injury of vascular wall, perivascular edema and perivascular hemorrhage $(P<0.05)$ (see Table 1).

\subsection{Electron Microscope Observation}

In Control group, tight junction disappeared among endothelial cells under electron microscope observation and cell membrane was incomplete, with partial or complete drop or even necrosis, cytoplasm dissolved. Rough endoplasmic reticulum expanded and the mitochondria of cytoplasm were so highly swell that the ridge fractured or even disappeared, shaped empty bubble degeneration (see Figure 2(a)). In Gelatum group, intravascular cortex was not complete and the matrix was exposed while some mitochondria were blurry, the vascular cavity of the endoplasmic reticulum was expanded and intercellular substance was slightly edematous (see Figure 2(b)). In Gel heated group, vascular endothelial cell was complete, mitochondrial crista was clear, intercellular substance was slightly edematous, endoplasmic reticulum was slightly expanded and the junction of endothelial cells was compact (see Figure 2(c)).

\section{DISCUSSION}

$20 \%$ mannitol is a medicine of high osmotic dehydra 
Table 1. Comparison among the veins and perivascular tissues of three groups $(n=8)$.

\begin{tabular}{|c|c|c|c|c|c|c|c|c|c|c|c|c|c|c|c|c|c|c|c|c|c|c|c|c|}
\hline \multirow[b]{2}{*}{ Groups } & \multicolumn{4}{|c|}{$\begin{array}{l}\text { Injury of vascular } \\
\text { wall }\end{array}$} & \multicolumn{4}{|c|}{$\begin{array}{l}\text { Vascular } \\
\text { dilatation }\end{array}$} & \multicolumn{4}{|c|}{$\begin{array}{l}\text { Perivascular } \\
\text { edema }\end{array}$} & \multicolumn{4}{|c|}{$\begin{array}{l}\text { Perivascular } \\
\text { hemorrhage }\end{array}$} & \multicolumn{4}{|c|}{$\begin{array}{c}\text { Infiltration of } \\
\text { inflammatory cell }\end{array}$} & \multicolumn{4}{|c|}{$\begin{array}{c}\text { Formation of } \\
\text { thrombus }\end{array}$} \\
\hline & - & + & $\begin{array}{l}+ \\
+\end{array}$ & $\begin{array}{l}+ \\
+ \\
+\end{array}$ & - & + & $\begin{array}{l}+ \\
+\end{array}$ & $\begin{array}{l}+ \\
+ \\
+\end{array}$ & - & + & $\begin{array}{l}+ \\
+ \\
\end{array}$ & $\begin{array}{l}+ \\
+ \\
+\end{array}$ & - & + & $\begin{array}{l}+ \\
+\end{array}$ & $\begin{array}{l}+ \\
+ \\
+\end{array}$ & - & + & $\begin{array}{l}+ \\
+ \\
\end{array}$ & $\begin{array}{l}+ \\
+ \\
+\end{array}$ & - & + & $\begin{array}{l}+ \\
+\end{array}$ & $\begin{array}{l}+ \\
+ \\
+\end{array}$ \\
\hline Control & 0 & 2 & 4 & 2 & 0 & 2 & 3 & 3 & 0 & 2 & 3 & 3 & 0 & 3 & 3 & 2 & 0 & 4 & 3 & 1 & 3 & 3 & 2 & 0 \\
\hline Gelatum & 2 & 4 & 2 & 0 & 4 & 3 & 1 & 0 & 2 & 3 & 3 & 0 & 3 & 3 & 2 & 0 & 4 & 2 & 2 & 0 & 6 & 1 & 1 & 0 \\
\hline Gel heated & 6 & 2 & 0 & 0 & 5 & 2 & 1 & 0 & 5 & 2 & 1 & 0 & 6 & 1 & 1 & 0 & 5 & 2 & 1 & 0 & 6 & 2 & 0 & 0 \\
\hline$\chi^{2}$ value $^{\mathrm{a}}$ & \multicolumn{4}{|c|}{6.000} & \multicolumn{4}{|c|}{15.000} & \multicolumn{4}{|c|}{5.000} & \multicolumn{4}{|c|}{6.000} & \multicolumn{4}{|c|}{14.500} & \multicolumn{4}{|c|}{18.000} \\
\hline Pvalue $^{a}$ & \multicolumn{4}{|c|}{$0.021^{*}$} & \multicolumn{4}{|c|}{0.523} & \multicolumn{4}{|c|}{$0.023^{*}$} & \multicolumn{4}{|c|}{$0.021^{*}$} & \multicolumn{4}{|c|}{0.461} & \multicolumn{4}{|c|}{1.000} \\
\hline$\chi^{2}$ value $^{\mathrm{b}}$ & \multicolumn{4}{|c|}{4.500} & \multicolumn{4}{|c|}{1.000} & \multicolumn{4}{|c|}{5.500} & \multicolumn{4}{|c|}{6.000} & \multicolumn{4}{|c|}{6.000} & \multicolumn{4}{|c|}{6.000} \\
\hline Pvalue $^{\mathrm{b}}$ & \multicolumn{4}{|c|}{$0.022^{*}$} & \multicolumn{4}{|c|}{$0.005^{*}$} & \multicolumn{4}{|c|}{$0.037^{*}$} & \multicolumn{4}{|c|}{$0.044^{*}$} & \multicolumn{4}{|c|}{$0.042^{*}$} & \multicolumn{4}{|c|}{$0.021^{*}$} \\
\hline$\chi^{2}$ value $^{c}$ & & & & & & 0.5 & & & & & & & & 0.0 & 00 & & & & & & & & 00 & \\
\hline Pvalue $^{c}$ & & & & & & 0.0 & & & & & & & & 0.0 & $2 *$ & & & & & & & 0.0 & & \\
\hline
\end{tabular}

Footnotes: ${ }^{a}$ the difference of injure degree between the Gelatum group and the Gel heated group; ${ }^{b}$ the difference of injure degree between the Control group and the Gelatum group; ${ }^{c}$ the difference of injure degree between the Control group and the Gel heated group, ${ }^{*} P<0.05$.

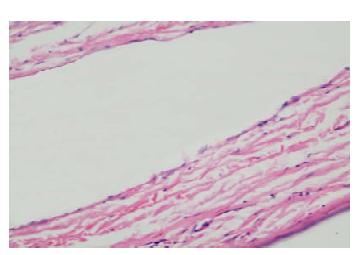

(a)

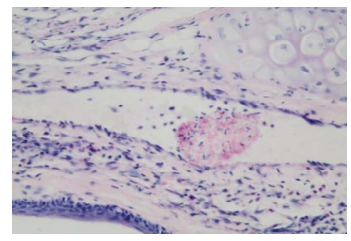

(c)

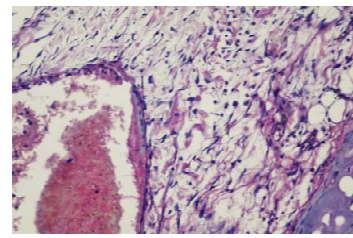

(b)

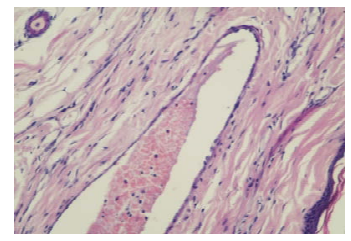

(d)
Figure 1. Histopathological changes of the veins on three groups, H-E, 400×. (a) Health group; (b) Control group; (c) Gelatum group; (d) Gelatum and heated mannitol group.

tion which needs to be rapidly infused into the body in order to produce better dehydration effect in the using process. While the intravenous osmotic pressure of local quickly increases when it is infused fast, and also leads to the increasing of osmotic pressure of local tissue dehydration of intravenous endothelial cells, the gathering of platelets and the releasing of prostaglandin $E_{1}$ and $E_{2}$, which increase the permeability of the venous wall and cause the infiltration and inflammation changes of leucocyte. At the same time, histamine of the local is released, which results in vein degeneration and lumen occlusion. Due to the short half-life period of mannitoland fast excretion, mannitol usually needs to be used multiple times a day in clinics in order to maintain the dehydration function, but the vessels can be damaged critically. The studies showed that the injury of the veins

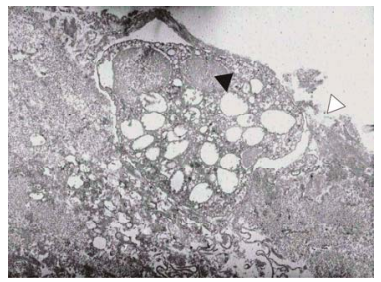

(a)

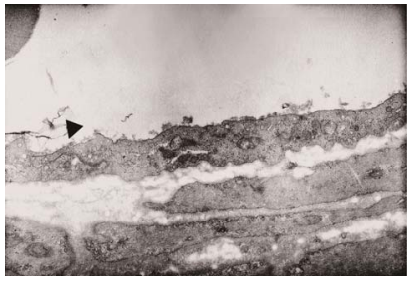

(b)

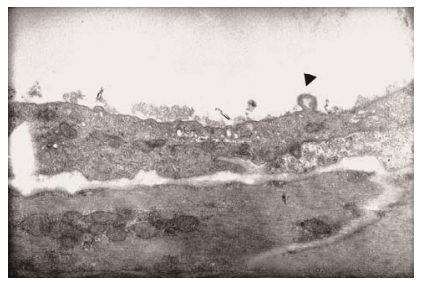

(c)

Figure 2. Ultrastructure of of the veins on three groups. (a) Membrane of endothelial on control group cell came off $(\triangle)$, the edema and cavitation bubble of mitochondria $(\boldsymbol{\Delta}), 6000 \times$; (b) Membrane of endothelial cell on intervention gelatum group was defective, the substrate was exposed $(\boldsymbol{\Delta}), 15,000 \times$; (c) Function of phagocyte on intervention gelatum and heated mannitol group was completed $(\boldsymbol{\Delta}), 15,000 \times$.

and perivascular tissue on the Control group is serious than that on Health group. From the observation of ultrastructure, we can see that serious pathological changes occurred in the control, such as falling off and necrosis of the membrane of the vascular endothelial cells, highly swelling of mitochondria, cristae dissolution or cavitation bubble of mitochondria. This shows that chemical phlebitis caused by $20 \%$ mannitol solution (we took samples from the area $0.5 \mathrm{~cm}$ away from the vein detained needle pinpoint, centripetal to the pinpoint, which 
avoided the happening area of mechanical phlebitis) can make the vein walls completely defunct and even cause irreversible damage. Table 1 shows that in control group the rate of phlebitis after infusing mannitol for two days is $100 \%$, which is much higher than that reported by literature [6]. Rabbit ears are made up of cartilage whose mobility is large and difficult to control. At the same time, the veins are fine, so the mechanical damage of the blood vessels is heavy. It indicates that joint parts should be avoided when venous indwelling trocar is used in clinics and the patients should be informed to limit the activity of the podosoma, as described earlier [7]. On the other hand, thinner venous indwelling trocar is suggested to be used in order to reduce vascular stimulation caused by the venous indwelling trocar so as to cut down the incidence of mechanical phlebitis, but other study [8] has shown there was no significant difference between the occurrence of phlebitis and catheter size. The chief reason for this probably is race.

Compound aescine gel is made by aescine and diethylamine salicylate. Aescine can play a seal role to capillary wall by reducing the quantities of capillary wall holes and the diameter of capillary, it can reduce the liquid from the capillaries to the tissue so that the edema is lightened. Diethylamine salicylate can decrease a series of symptoms caused by inflammation because it inhibits synthesis and release of prostaglandin and other inflammatory mediators and antagonizes the permeability increase of capillary and lymphatic vessels caused by inflammatory mediators [9]. Research shows that comparison of injury degree differences of vein and perivascular tissues between Gelatum group and Control group had statistical significance $(P<0.05)$. The ultrastructure of Gelatum group shows that endothelial cells had a slight damage. Parts of mitochondria are clear. This indicates that compound aescine gel can reduce the incidence of phlebitis and alleviate the damage of blood vessels. It plays an obviously protective role in the vein vessels. As the matrix of compound aescine gel contains poly ethylene glycol-6 octylic acid glyceride, it is not easy to cause the dry of skin when externally applicated to the skin, and it can infiltrate subcutaneous tissue quickly and completely, so that the drug can play effect for a long time.

$20 \%$ mannitol is a kind of supersaturated solution, tiny crystal separates out with the decrease of temperature, adverse reactions may occur because of the embolization caused by foreign body easily formed after infusion of mannitol into the vein. The theoretical principle of keeping mannitol solution being maintained at a temperature of $35^{\circ} \mathrm{C}-37^{\circ} \mathrm{C}$ for injection is that rabbit's core temperature changes in the range of $38.0^{\circ} \mathrm{C}-39.5^{\circ} \mathrm{C}[10]$ making solution heated to $35^{\circ} \mathrm{C}-37^{\circ} \mathrm{C}$ won't induce the scald of tunicae intima vasorum and blood cell degen- eration; The number of particles in mannitol solution which is heated to $35^{\circ} \mathrm{C}$ is significantly reduced $[11,12]$, which can prevent a large number of particles in a short period of time from entering the vein, stirring up thrombosis and then leading to local blocking, insufficiency of blood supply, edema and inflammation caused by tissue hypoxia; At the same time, heated mannitol solution can make local blood vessels dilate, accelerate the flow of blood, thereby preventing macromolecular material depositing on the walls of blood vessels and helping to reduce vascular stimulation and vascular injury. Table 1 shows that comparison of injury degree differences of vein and perivascular tissues between Gel heated group and Control group has statistical significance $(P<0.05)$, the injury of vascular wall, perivascular edema and perivascular hemorrhage in Gel heated group mitigated significantly compared with the Gelatum group. Phenomena such as: integrity of vascular endothelial cell, complete function of phagocytic vesicle, clear description of mitochondrial cristae and so on. Under the electron microscopic, the observations of Gel heated group indicate the combined intervention of injecting heated mannitol solution and external application of gel can better prevent phlebitis and reduce the direct damage on vascular endothelial cells. Research of Kaiyu Pan [13] holds that vitamin $\mathrm{C}$ can effectively inhibit phlebitis caused by $20 \%$ mannitol solution. The research results from gene chip technology support that vitamin $\mathrm{C}$ can restrain apoptosis led by $20 \%$ mannitol solution. And in Control group of this experiment, the result of electron microscope research shows that many pathological changes are very similar to the phenomena of cell apoptosis. For example, the tight junction of the endothelial cells disappears and cell membrane is incomplete while the crest of mitochondria dissolves or disappears. Therefore, proper predictions can be drawn that compound of reparilgel $\mathrm{N}$ can restrain cell apoptosis induced by $20 \%$ mannitol solution, which provides scientific basis for clinical application.

\section{RELEVANCE TO CLINICAL PRACTICE}

From observations of the macroscopic and microscopic aspects, it can be found that $20 \%$ mannitol solution infused by venous indwelling trocar has a serious damage to the blood vessels, but compound aescine gel coated on local skin can prevent the occurrence of phlebitis or reduce the incidence of phlebitis and also reduce the injury degree of the blood vessels, which provides theoretical basis for the clinical protection of intravenous infusion vessels. Research of Pan Ky [14] holds that vitamin C can effectively inhibit phlebitis caused by $20 \%$ mannitol solution. The research results from gene chip technology support that vitamin $\mathrm{C}$ can restrain apoptosis 
led by $20 \%$ mannitol solution. In consideration of this research, compound of compound aescine gel can restrain cell apoptosis induced by $20 \%$ mannitol solution, which provides scientific basis for clinical application. The domestic and foreign research shows that there has a close relationship between the diameter of venous indwelling trocar and formation of thrombus, which is the longer of the diameter, the higher incidence of phlebitis $[15,16]$. Our research indicates that it is necessary to avoid the joints and minimize physical activity of the area with venous indwelling trocar in order to reduce the incidence of phlebitis. Once the findings were applicated to clinical practice of nursing, it can extend the trocar indwelling time, relieve the pain of patients and play an important role in improving the quality of service on venous transfusion.

\section{CONCLUSION}

Observations from the macroscopic and microscopic aspects can draw a conclusion that $20 \%$ mannitol solution infused by venous indwelling trocar has a serious damage to the blood vessels. Compound aescine gel can prevent the occurrence of phlebitis or reduce the incidence of phlebitis. It can also reduce the injury degree of the blood vessels, extend the trocar indwelling time and exert the advantages of the venous indwelling trocar. The concerted intervention of external application of compound aescine gel and infusion of the heated $20 \%$ mannitol solution (maintained at $35^{\circ} \mathrm{C}-37^{\circ} \mathrm{C}$ ) can produce better effect on protecting vessels.

\section{ACKNOWLEDGEMENTS}

The source of funding for the study was supported by The Education Department of Henan Province. We are indebted to Xinhua Cai of Department of Histology and Embryology for expert help in picture analyses and suppliers of materials-University Key Laboratory of Tissue Regeneration in Henan Province.

\section{REFERENCES}

[1] Talagakis, V., Kahn, S.R., Libman, M. and Blostein, M. (2002) The epidemiology of peripheral vein infusion thrombophlebitis: A critical review. The American Journal of Medicine, 113, 146-151. doi:10.1016/S0002-9343(02)01163-4

[2] Lu, B.Q., Wang, H.P., Chen, W., et al. (2009) Phlebitis in cancer patients treated with chemotherapy and its correlates. Nursing Journal of Chinese People's Liberation Army, 26, 5-7.
[3] Xin, Y.F., Wang, L.P., Qiao, L.Q., et al. (2000) Experimental study of the damage of mannitol at different temperatures to the ear-vein of rabbits. Nursing Journal of Chinese People's Liberation Army, 17, 1-2.

[4] Sun, C.H. (2008) Observation to vascular protection on infusing mannitol. Journal of Emergency in Traditional Chinese Medicine, 17, 1029-1030.

[5] Ren, X.D., Qu, Z.P., Yang, X., et al. (1998) The effect of mannitol on peripheral veins in the rabbit. Chinese Journal of Nursing, 33, 68-70.

[6] Chen, L.F., Xu, C. and Zhou, W.M. (2010) The observation on pulse type flushing tube combined with magnesium sulfate wet compress to prevent phlebitis' effect. Shanghai Journal of Preventive Medicine, 22, 214-215.

[7] Gorski, L.A. and HHCNS-BC (2004) Speaking of standards. The Art and Science of Infusion Nursing, 32, 125126. doi:10.1097/NAN.0b013e3181a2835a

[8] Uslusoy, E. and Mete, S. (2008) Predisposing factors to phlebitis in patients with peripheral intravenous catheters: A descriptive study. Journal of the American Academy of Nurse Practitioners, 20, 172-180. doi:10.1111/j.1745-7599.2008.00305.x

[9] Jin, H. (2001) The function and application of Reparilgel N. Chinese New Drugs Journal, 6, 128.

[10] Xiang, Q.J., Li, C.S. and Hu, Y., et al. (2009) Effect of the different basal body temperature of rabbits on pyrogen test results. Progress in Microbiology and Immunology, 37, 28-31.

[11] Malek, A.M., Goss, G.G., Jiang, L., et al. (1998) Mannitol at clinical concentration activates multiple signaling pathways and induces apoptosis in endothelial cells. Stroke, 29, 2631-2640. doi:10.1161/01.STR.29.12.2631

[12] Sun, W.X., Lin, L.H., Cao, C.H., et al. (2009) The toxic effects and corresponding countermeasures of mannitol. Chinese Journal of Modern Drug Application, 3, 142143.

[13] Pan, K.Y., Shang, S.Q., Dai, X.N., et al. (2006) An experiment on inhibitive affection of vitamin $\mathrm{C}$ on the apoptosis of vascular endothelial cell induced by mannitol. Journal of Pediatric Pharmacy, 12, 1-3.

[14] Pan, Ky., Shen, M.P., Ye, Z.H., et al. (2006) Inhibitive effects of anti-oxidative vitamins on mannitol-induced apoptosis of vascular endothelial cells. Journal of Zhejiang University. Science B, 7, 825-829. doi:10.1631/jzus.2006.B0825

[15] Peng, C.X. (2000) Application of two type vein detained needle in general surgery infusion. Journal of Nursing Science, 15, 617.

[16] Grove, J.R. and Pevec, W.C. (2003) Venous thrombosis related to peripherally inserted central catheters. Journal of Vascular and Interventional Radiology, 1, 83. 